\title{
VARIAÇÕES CLIMÁTICAS NA PRECIPITAÇÃO NO SUL DO BRASIL NO CLIMA PRESENTE E FUTURO
}

\author{
JOSSANA CEOLIN CERA ${ }^{1}$, SIMONE EROTILDES TELEGINSKI FERRAZ ${ }^{2}$ \\ ${ }^{1}$ Universidade Federal de Santa Maria (UFSM), Departamento de Fitotecnia, Santa Maria, RS, Brasil, \\ ${ }^{2}$ UFSM, Departamento de Física, Santa Maria, RS, Brasil. \\ jossana.cera@gmail.com, simonetfe@gmail.com
}

Enviado Fevereiro de 2013 - Aceito 26 de Junho de 2014.

\begin{abstract}
RESUMO
Em meio ao grande destaque que o clima e suas mudanças vêm apresentando nos últimos tempos, viu-se a importância de uma análise das tendências de precipitação para a compreensão dos possíveis impactos que as mudanças no clima possam ocasionar na sociedade em geral. Portanto, este trabalho apresenta uma análise da tendência de precipitação para o Rio Grande do Sul, para três conjuntos de dados, com uma comparação entre dados de precipitação observada e dados simulados pelo Modelo Regional RegCM3 e uma análise de tendência para um cenário de clima futuro. Esta análise foi realizada através de diferentes testes estatísticos, que calcularam as tendências de precipitação para os dados de precipitação observada e para os dados do Modelo RegCM3 para o mesmo período, 1982 a 2006, e para os dados do modelo para o clima futuro no período de 2070 a 2086. Avaliando a porcentagem de eventos secos e chuvosos obtidos dos dados observados (PREC_LA), nota-se que os modos na banda sinótica contribuem mais para os eventos secos do que para os eventos chuvosos. Já na banda intrassazonal, há mais contribuição para eventos chuvosos do que para eventos secos. $\mathrm{Na}$ banda interanual, os modos analisados contribuem igualmente para eventos secos e chuvosos. No entanto, quando observa-se os eventos nos dados simulados (PREC_RE), verifica-se que o modelo não conseguiu reproduzir os resultados observados.
\end{abstract}

Palavras-chave: Tendência; Cenários Futuros; Precipitação.

\begin{abstract}
CLIMATE VARIABILITY IN SOUTHERN BRAZIL PRECIPITATION IN PRESENT AND FUTURE CLIMATE

Climate and its changes have been showing great prominence lately. In this way, it is important to analyze the precipitation trends for the understanding of the potential impacts that changes in climate may result in the society. This paper presents an analysis of the precipitation trend in Rio Grande do Sul, for three sets of data, with a comparison of the observed precipitation data and simulated data by RegCM3 Regional Model, as well as an analysis of the trend in a future climate scenario. This analysis was performed using different statistical tests, which calculated the of precipitation trends for the observed precipitation data, for the RegCM3 model data for the same period, 1982-2006, and for the 2070 to 2086 future climate modeled data. Evaluating the percentage of dry and wet events obtained from the observed data (PREC_LA), it was found that the modes in the synoptic band contribute greatly to dry events than to wet events. Yet in the intraseasonal band there is more contribution to the wet than to the dry events. The analyzed modes in the interannual band contribute equally to dry and wet events. However, when we observed the events in the simulated data (PREC_RE), the model failed to reproduce the observed results.
\end{abstract}

Keywords: Trend; Future Scenarios; Precipitation. 


\section{INTRODUÇÃO}

A Região Sul do Brasil tem grande parte de sua economia associada ao agronegócio e as características do clima e suas variações podem determinar anos favoráveis ou desfavoráveis para a produção agrícola e criação de animais, entre outros. Deste modo, a produção agropecuária do Rio Grande do Sul tem o clima como principal fator responsável por quebras de safras e, consequentemente, diminuição de lucros. Neste sentido, buscou-se utilizar ferramentas apropriadas para fazer um estudo mais racional sobre as tendências do clima, a fim de minimizar perdas e conhecer o clima da região com maior clareza e detalhes.

O Rio Grande do Sul situa-se em uma região latitudinal favorável à atuação de diversos fenômenos meteorológicos que afetam o tempo e o clima do estado como, por exemplo, as frentes frias e o fenômeno El Niño. Considerando os fenômenos de escala sinótica, têm-se as frentes frias que passam regularmente pelo estado favorecendo a boa distribuição espacial e temporal das chuvas, conforme mostrado em Cera e Ferraz (2007). Fenômenos de escala intrassazonal como a Oscilação de Madden e Julian (OMJ) também influenciam indiretamente a precipitação no estado (Ferraz, 2004). Na escala global, observam-se as influências remotas dos Oceanos Atlântico e Pacífico no regime das chuvas no Sul do Brasil (Grimm et al, 1998; Cardoso, 2005; Grimm, 2009; Firpo, 2012). Neste padrão de baixa frequência, têm-se os fenômenos El Niño e La Niña que são caracterizados, respectivamente, pelo aquecimento e resfriamento anômalo das águas do Oceano Pacífico Equatorial central e leste. Atuando juntamente com estes dois fenômenos, tem-se o Índice de Oscilação Sul (IOS) que representa um fenômeno de grande escala caracterizado pela diferença da Pressão ao Nível do Mar entre o Pacífico Central (Taiti) e o Pacífico Oeste (Darwin/Austrália), causando o enfraquecimento ou intensificação dos ventos alísios sobre o Oceano Pacífico Equatorial. O acoplamento destes fenômenos, oceânico e atmosférico, nesta região é chamado de El Niño Oscilação Sul (ENOS). A variação anômala destas componentes (El Niño/La Niña e IOS) mostra duas fases opostas do ENOS, que em média tem periodicidade de quatro a sete anos e duração média de doze a dezoito meses. Grimm (2003) em seus estudos verificou a influência do El Niño na circulação monçônica de verão, concluindo que as anomalias de precipitação são favorecidas pela perturbação na circulação das Células de Walker e Hadley sobre o Oceano Pacífico Leste e América do Sul, e pelos trens de ondas de Rossby sobre o sul da América do Sul.

Além destes, modos de variabilidade de baixíssima frequência, como a Oscilação Decenal do Pacífico (ODP), possuem importantes teleconexões climáticas. Tal como o fenômeno ENOS, nas suas fases quente e fria, a ODP pode influenciar a região nordeste do estado do Rio Grande do Sul (Cera et al., 2009). Este fenômeno de escala decenal superposto a um fenômeno de escala interanual, como o ENOS, pode alterar o efeito deste em algumas regiões do globo, inclusive na América do Sul (Kayano e Andreoli, 2009).

Além da variabilidade natural que afeta o clima na América do Sul há indícios de possíveis alterações no clima relacionadas a efeitos antropogênicos. Em 1990, no primeiro relatório do Painel Intergovernamental de Mudanças Climáticas (IPCC, na sigla em Inglês do Intergovernmental Panel on Climate Change), sugeriu-se um aumento da temperatura global média entre $0,15^{\circ} \mathrm{C}$ e $0,3^{\circ} \mathrm{C}$ por década de 1990 a 2005 . Em relação à precipitação, os resultados não são conclusivos, pois dependem dos modelos empregados nas previsões e das regiões consideradas (Marengo, 2009).

Este estudo visa determinar, de forma mais abrangente, as mudanças climáticas da precipitação que possam afetar a agricultura, a população e a economia do Rio Grande do Sul. As variáveis analisadas são decorrentes de integrações de longo prazo para caracterizar as condições climáticas do Rio Grande do Sul em diferentes cenários.

Neste sentido, o estudo tem por objetivo determinar as tendências de precipitação para o clima presente e futuro do Rio Grande do Sul, bem como encontrar os principais padrões de variabilidade da precipitação.

\section{MATERIAIS E MÉTODOS}

Utilizaram-se dados diários de precipitação de estações pluviométricas localizadas no Rio Grande do Sul, estes dados observados do clima presente (a partir daqui chamados PREC LA) foram obtidos do conjunto de dados de Liebmann e Allured (Liebmann e Allured, 2005) que estão combinados na forma de campos diários de precipitação, com espaçamento de grade de $1^{\circ} \mathrm{X} 1^{\circ}$ e $2,5^{\circ} \mathrm{X} 2,5^{\circ}$ para o período de $1940-2008$. Neste estudo utilizaram-se os dados com espaçamento de grade $1^{\circ} \mathrm{X}$ $1^{\circ}$ no período de 1982 a 2006, pois este período e espaçamento são comuns aos dados do modelo climático regional que foi utilizado nas simulações.

Os dados observados de precipitação foram comparados com os dados simulados para o mesmo período pelo Modelo Regional RegCM3, do ICTP (International Centre for Theoretical Physics), descrito em Pal et al. 2000, a fim de verificar se os dois conjuntos de dados são coerentes, ou seja, verificar se os dados da simulação possuem similaridades com os dados observados (este conjunto de dados será chamado de PREC_RE). Os dados utilizados para a análise do clima futuro (PREC_FU) são oriundos das rodadas climáticas regionais do Projeto PROBIO (Projeto de Conservação e Utilização 
Sustentável da Biodiversidade Biológica Brasileira, dados disponíveis para 2070-2086), que teve como objetivo fornecer informação técnico-científica sobre as possíveis mudanças climáticas sobre o território brasileiro (Marengo, 2006).

Para testar a homogeneidade das séries de dados usou-se o teste Run (ou de Sequência), que é um teste não paramétrico (Back, 2001; Minuzzi et al., 2010). O teste Run consiste na contagem do número de oscilações dos valores acima e abaixo da mediana, em uma série naturalmente ordenada. Os valores observados devem ser testados para verificar se estão dentro da faixa considerada normal, por isso a hipótese nula consta que a distribuição das sequências ocorre normalmente, dessa forma a amostra ocorre aleatoriamente (Back, 2001). Para analisar se há tendência em séries históricas fez-se uso de dois testes, o teste Mann-Kendall e o teste Pettit. O teste Mann-Kendall foi inicialmente proposto por Sneyers (1975) e considera que, na hipótese de estabilidade de uma série temporal, a sucessão de valores ocorre de forma independente e a distribuição de probabilidade deve permanecer sempre a mesma (Back, 2001). $\mathrm{O}$ teste de Mann-Kendall é o método mais apropriado para analisar mudanças significativas em séries climatológicas e permite também a detecção e localização aproximada do ponto inicial de determinada tendência (Siegel, 1975; Back, 2001). O teste de Pettit é um teste não paramétrico que utiliza uma versão do teste de Mann-Whitney, no qual é testado se as amostras são da mesma população. Esta estatística localiza o ponto de mudança ou ruptura brusca na série temporal (Back, 2001).

Para a determinação dos padrões de precipitação filtrou-se os dados a fim de reter a variabilidade sinótica e a variabilidade intrassazonal das séries de dados utilizando-se o filtro de Lanczos (Duchon, 1979) para as bandas de 2 a 20 dias e 20 a 100 dias, respectivamente. Para a variabilidade interanual, foi calculada a média anual da precipitação. Após a filtragem, os dados foram analisados através da Análise de Componentes Principais (ACP) com rotação de modos, para caracterizar as variabilidades nas três bandas durante as quatro estações do ano.

Realizou-se também uma comparação entre as séries das Componentes Principais para determinar os eventos secos e chuvosos durante o período de análise. Para isto, utilizou-se a série temporal reconstruída dos modos rotacionados para as três bandas de frequência e para os três períodos analisados. Para a escala sinótica e intrassazonal foram considerados como eventos chuvosos ou secos aqueles que possuíssem três ou mais dias consecutivos com valores de componentes principais maiores ou iguais a $+1,2$ desvio padrão ou menores ou iguais a $-1,2$, respectivamente. Para a escala interanual foram considerados os anos com componentes principais maiores ou iguais a $+1,2$ desvio padrão ou menores ou iguais a $-1,2$, conforme a metodologia utilizada por Paegle e Mo (1997).
Cabe ressaltar que os dados de clima futuro foram analisados para um período menor de anos, dezessete anos (2070-2086, disponível do projeto PROBIO), contra vinte e cinco dos dois outros conjuntos (1982-2006).

\section{RESULTADOS E DISCUSSÕES}

Para validação dos dados do modelo foi realizada uma análise do conjunto de dados observados e simulados pelo modelo RegCM3, para o período de dados de 1982 a 2006. Posteriormente, com base na climatologia de 1960 a 1990 fez-se o prognóstico do clima futuro para o período de 2070 a 2086.

A hipótese nula do teste Run é de que a distribuição dos dados ocorre normalmente e que a amostra é aleatória, sendo testada pela estatística Z (Tabela 1). Para rejeitar esta hipótese nula com nível de significância melhor que $5 \%, Z$ deve ser menor que $-1,96$ ou maior que 1,96 e, a probabilidade de obter um valor da estatística do teste como o observado, deve ser menor que 0,05 . Como isto não ocorre com nenhuma das séries de precipitações anuais, conclui-se que a hipótese nula é verdadeira e as séries de dados são homogêneas e, portanto, podem ser usadas em estudos mais detalhados que envolvam análises de tendência climática (Tabela 1). Através da análise do teste Pettit, observou-se o ano da quebra de tendência das séries de precipitação usadas neste trabalho, em que a maioria dos anos estiveram compreendidos na fase quente do fenômeno ENOS (anos de El Niño). O Teste Pettit (K) é um teste não paramétrico, que faz parte de uma série de testes de homogeneidade, no qual a hipótese nula é que a série temporal é homogênea em tempos

Tabela 1 - Teste Run $(Z, p)$ e o Teste Pettit (K) aplicados às séries de dados de precipitação anual do Rio Grande do Sul, no período de 1982 a 2006.

\begin{tabular}{|c|c|c|}
\hline Ponto & $\mathbf{Z}$ & $\mathbf{K}$ \\
\hline $30^{\circ} \mathrm{S} 57^{\circ} \mathrm{W}$ & $-1,424$ & 2003 \\
\hline $30^{\circ} \mathrm{S} 56^{\circ} \mathrm{W}$ & 0,212 & 2003 \\
\hline $30^{\circ} \mathrm{S} 53^{\circ} \mathrm{W}$ & 0,212 & 1987 \\
\hline $31^{\circ} \mathrm{S} 56^{\circ} \mathrm{W}$ & $-0,196$ & 2003 \\
\hline $32^{\circ} \mathrm{S} 54^{\circ} \mathrm{W}$ & $-0,196$ & 2003 \\
\hline $32^{\circ} \mathrm{S} 53^{\circ} \mathrm{W}$ & $-1,424$ & 2002 \\
\hline $32^{\circ} \mathrm{S} 52^{\circ} \mathrm{W}$ & $-1,424$ & 1987 \\
\hline $33^{\circ} \mathrm{S} 53^{\circ} \mathrm{W}$ & $-1,424$ & 1987 \\
\hline $29^{\circ} \mathrm{S} 57^{\circ} \mathrm{W}$ & 0,212 & 2003 \\
\hline $29^{\circ} \mathrm{S} 56^{\circ} \mathrm{W}$ & $-0,605$ & 1987 \\
\hline $29^{\circ} \mathrm{S} 53^{\circ} \mathrm{W}$ & 0,212 & 1987 \\
\hline $29^{\circ} \mathrm{S} 51^{\circ} \mathrm{W}$ & 0,622 & 2003 \\
\hline $29^{\circ} \mathrm{S} 50^{\circ} \mathrm{W}$ & 0,622 & 1987 \\
\hline $28^{\circ} \mathrm{S} 55^{\circ} \mathrm{W}$ & 0,212 & 1987 \\
\hline $28^{\circ} \mathrm{S} 54^{\circ} \mathrm{W}$ & $-0,196$ & 1998 \\
\hline $28^{\circ} \mathrm{S} 53^{\circ} \mathrm{W}$ & 0,622 & 2002 \\
\hline $28^{\circ} \mathrm{S} 52^{\circ} \mathrm{W}$ & 0,622 & 1998 \\
\hline $28^{\circ} \mathrm{S} 51^{\circ} \mathrm{W}$ & 0,622 & 2002 \\
\hline
\end{tabular}


distintos. Além disso, é possível verificar se houve ruptura da série e em que ponto ela ocorreu (Tabela 1). Nesse caso, o ano de ruptura da série de precipitação é dado pelo valor de K, conforme a Tabela 1, ao nível de significância de 0,05.

Estes dois testes mostraram resultados contraditórios, pois enquanto o teste Run indicou que as séries são homogêneas, o teste de Pettit indicou uma ruptura (ou seja, não homogeneidade). Para confirmar qual hipótese estava correta, realizou-se mais um teste: o de Mann-Kendall. Como será observado nos resultados a seguir, este teste confirmou que há tendência nos dados e, portanto, eles não são homogêneos. Cabe ressaltar que a aplicação de apenas um teste, muitas vezes, pode indicar resultados incorretos, como foi observado.

Analisando as tendências das séries de dados observados obtidas através do teste Mann-Kendall (Figura 1a) encontrou-se tendência de aumento na precipitação em grande parte do Rio Grande do Sul, para o período de 1982 a 2006, com tendências significativas ao nível de 99,9\% em alguns pontos, como no extremo norte e região central do Estado. Tendências negativas de precipitação ao nível de 90 e $99,9 \%$ foram observadas no extremo oeste e região litorânea do Estado e em parte da região norte. Estas tendências positivas estão de acordo com as encontradas em Haylock et al. (2006), que em seu estudo encontrou tendências positivas na precipitação sobre todo o Rio Grande do Sul, para o período de 1960 a 2000. Sansigolo e Kayano (2010) analisaram a tendência da precipitação para seis cidades no Rio Grande do Sul durante o período de 1913 a 2006 e seus resultados também mostraram uma tendência de aumento na precipitação, principalmente durante o verão.

Nos dados simulados pelo Modelo RegCM3 (Figura 1b) não foram constatadas tendências significativas, a não ser no sul do Estado, onde foram encontradas tendências de aumento na precipitação com significância de 90 e 99,9\%, o que está parcialmente de acordo com o observado no período, dando assim alguma confiabilidade ao Modelo Climático Regional em simular essas tendências de precipitação nesta região do Rio Grande do Sul.

Sabatini (2008) analisou a tendência da precipitação sazonal e verificou que apenas no verão houve tendências negativas sobre o Sul do Brasil, para as outras estações as tendências foram positivas. A tendência de aumento dos totais anuais de chuva se sobrepõe à tendência negativa de apenas uma estação do ano e concorda com as observações. Contudo, quanto ao verão, difere dos resultados de Sansigolo e Kayano (2010), baseados em séries observadas, que mostram tendência de aumento principalmente no verão.

Analisando a simulação dos dados para o clima futuro (Figura 1c) observou-se tendências significativas de aumento na precipitação (a 90\% de significância) para as regiões oeste, sul e central do estado. Estas tendências são semelhantes às encontradas para o período de 1982 a 2006, especialmente nas regiões central e sul. Esta predominância de tendência de aumento da precipitação está de acordo com as projeções do AR4 (IPCC, 2007) que indicaram aumento na precipitação para a Região Sul do Brasil.

A Análise de Componentes Principais, aplicada aos dados diários e anuais de precipitação observada e simulada pelo modelo, determinou os modos de variabilidade de precipitação que afetam o Rio Grande do Sul. Dos modos encontrados, verificou-se através da comparação do padrão espacial de cada um deles, que três modos se repetiam em algumas bandas de variabilidade de todos os conjuntos de dados e nas diversas estações do ano. Estes foram denominados Modo Inter (Figura 2a), Modo PR (Figura 2b) e Modo Sul (Figura 2c). O Modo Inter aparece na banda interanual e mostra valores mais intensos concentrados no Rio Grande do Sul (o nome foi escolhido por
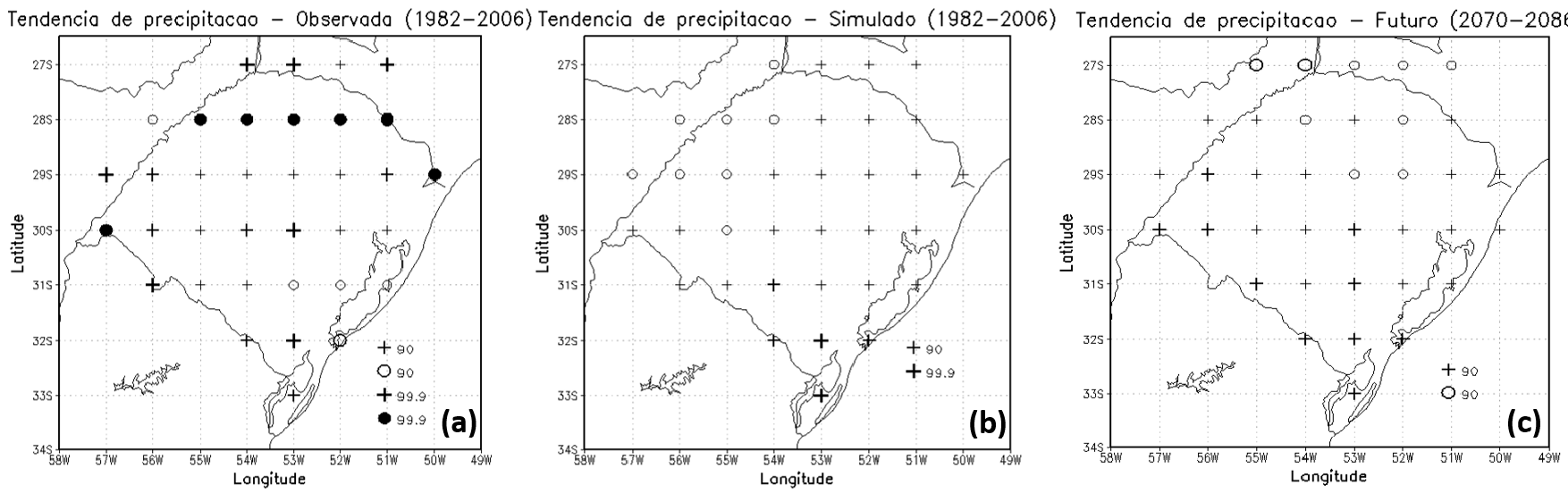

Figura 1 - Distribuição da tendência de precipitação obtidas através do teste Mann-Kendall: (a) observada - período de 1982 a 2006,(b) simulada - período de 1982 a 2006 e (c) simulada - período de 2070 - 2086. O sinal de '+' indica tendência positiva e o sinal de '• ou o' indica tendência negativa. Os sinais menores indicam as tendências sem significância (que não estão na legenda) e os maiores indicam as tendências com nível de confiança de 90 e $99,9 \%$. 
MODO INTER

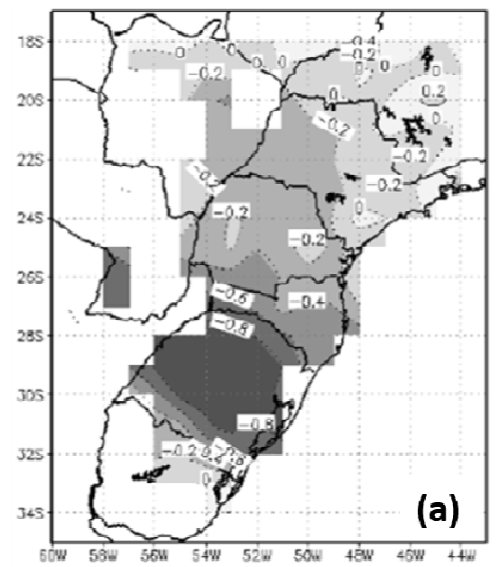

MODO PR

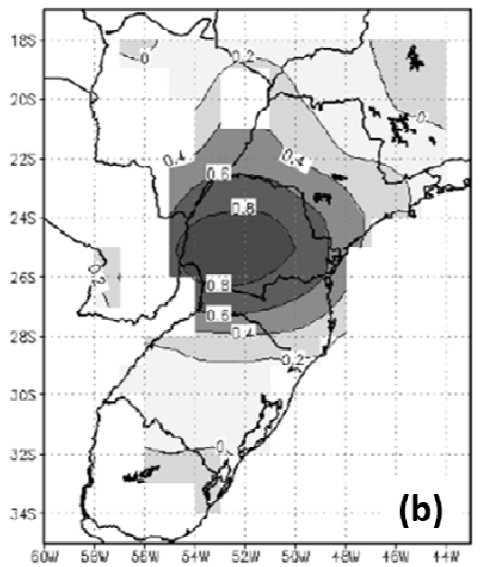

MODO SUL

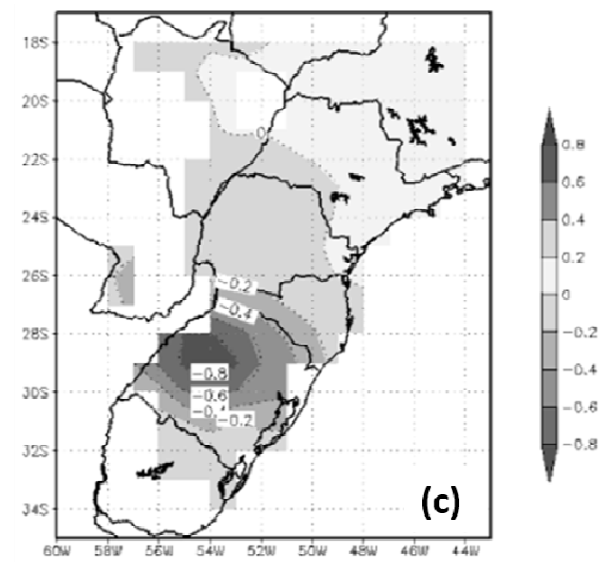

Figura 2 - Caracterização de três modos de variabilidade encontrados para a Região Sul do Brasil. (a) Modo Inter, (b) Modo PR e (c) Modo Sul. O Modo Inter foi obtido através de dados observados e de simulação na banda interanual, o Modo PR foi obtido também através de dados observados e de simulação nas bandas interanual, intrassazonal e sinótica e o Modo Sul foi encontrado para os mesmos dados anteriores nas bandas sinótica e intrassazonal. Estes três modos foram encontrados para as quatro estações do ano.

remeter à importância desta variabilidade na banda interanual). O Modo PR aparece nas três bandas (sinótica, intrassazonal e interanual) e indica que o núcleo do modo é sobre o Paraná, e o Modo Sul aparece na banda sinótica e intrassazonal e, como definido em Ferraz (2004) e Pampuch (2010), apresenta núcleo mais intenso no Rio Grande do Sul. O enfoque da análise a seguir se restringirá a estes três modos, independente deles terem a maior variância explicada, ou não.

A variabilidade interanual é caracterizada por ter influência de fenômenos de baixa frequência como o fenômeno ENOS (nomenclatura utilizada por James (1994)). Considerando os dados observados de clima presente (PREC_LA), o Modo Inter aparece com $15,3 \%$ da variância explicada no $1^{\circ}$ modo durante o verão. Para os dados simulados pelo modelo para o clima presente (PREC_RE), o Modo Inter só não se configurou na primavera, quando seu núcleo é deslocado para norte, sobre o Paraná. Já para as outras estações, apareceu como $2^{\circ}$ modo: no outono, com $13,6 \%$ da variância explicada; no inverno, com $15,6 \%$ e no verão, com $12,4 \%$. O Modo Inter, para o clima futuro (PREC_FU) não se configurou da mesma maneira que nos outros dois conjuntos de dados. Durante o outono e inverno se configurou com núcleo deslocado para o noroeste do Rio Grande do Sul no $4^{\circ}$ modo com rotação, com $6,1 \%$ e $10,4 \%$ da variância explicada, respectivamente. Durante a primavera apareceu no $3^{\circ}$ modo com rotação, com $14,4 \%$ e, no verão, apareceu no $1^{\circ}$ modo com $28,46 \%$ da variância explicada.

Cabe ressaltar que a variabilidade interanual tem significativa importância em parte da Região Sul (Grimm e Zilli, 2009). Em alguns modos observou-se precipitação com contribuições negativas mais ao sul e positivas na Região Sudeste, o que pode estar associado ao período mais seco na região Sudeste e mais chuvoso no extremo sul do país, ou vice-versa.

$\mathrm{Na}$ escala intrassazonal o Modo PR aparece bem configurado durante a primavera para os três conjuntos de dados (PREC_LA, PREC_RE e PREC_FU). Para os dados observados (PREC_LA) o Modo PR apareceu como $2^{\circ}$ modo rotacionado, durante a primavera, com $10,8 \%$ da variância e no $4^{\circ}$ modo durante o outono, com $9,1 \%$ da variância. Para os dados PREC_RE, o Modo PR apareceu no $1^{\circ}$ modo durante o inverno, primavera e verão $(7,3 \%, 7,9 \%$ e $6,2 \%$ da variância, respectivamente) e no $2^{\circ}$ modo com $6,9 \%$ da variância no outono. Já para os dados PREC_FU, o Modo PR foi encontrado no $1^{\circ}$ para o outono e primavera ( $11,7 \%$ e $11,1 \%$ da variância $)$ e no $3^{\circ}$ modo durante o verão com $8,5 \%$ da variância explicada.

$\mathrm{Na}$ escala sinótica, para os dados PREC_LA, o Modo Sul foi o $1^{\circ}$ modo com rotação durante a primavera $(7,6 \%$ da variância explicada). No verão, outono e inverno, o Modo Sul foi encontrado no $5^{\circ}$ modo com $4,1 \%, 4,3 \%$ e $5 \%$ da variância, respectivamente. Para os dados PREC_RE, o Modo Sul foi encontrado no $1^{\circ}$ modo durante o outono, inverno e primavera ( $3,9 \%, 3,6 \%$ e $5,1 \%$ da variância, respectivamente), exceto durante o verão. Para os dados PREC_FU, foi encontrado no $3^{\circ}$ modo para o outono, no $2^{\circ}$ modo para o inverno, no $4^{\circ}$ modo para a primavera e no $1^{\circ}$ modo para o verão, com $3,2 \%, 4,5 \%$, $2,5 \%$ e $3,2 \%$ da variância, respectivamente. O Modo Sul é mais perceptível em escalas de alta frequência, como a escala sinótica. Ferraz (2004) verificou que a variabilidade na banda sinótica é mais intensa no Sul do Brasil que a variabilidade intrassazonal, e talvez isto explique por que este modo fica bem definido na banda sinótica e num período em que a variabilidade sinótica é bem ativa (primavera). 
Com relação à quantidade de eventos secos e chuvosos, obtiveram-se a Tabela 2 e a Figura 3, que mostram a quantidade de eventos para cada estação do ano, para os três conjuntos de dados, além dos três modos de variabilidade encontrados nas três bandas de frequência. De maneira geral, percebe-se que os eventos, tantos secos como chuvosos, aparecem em maior número na banda intrassazonal, ou seja, o Modo Sul e o Modo PR contribuem com o maior número de eventos nas três séries temporais (PREC_LA, PREC_RE E PREC_FU), sendo o Modo Sul, na banda intrassazonal, o que mais contribui com os eventos.

Analisando os dados PREC_LA (Tabela 2 e Figuras 3a, 3b), observa-se que os modos Sul e PR, na banda sinótica, contribuem mais para eventos secos do que para eventos chuvosos. Já na banda intrassazonal, tais modos contribuem mais para eventos chuvosos do que para eventos secos. O Modo PR e Modo Inter na banda interanual possuem praticamente o mesmo número médio de eventos secos e chuvosos.

Já para os dados PREC_RE, nota-se que os modos Sul e PR na banda sinótica contribuem mais para eventos chuvosos do que para eventos secos, enquanto na banda intrassazonal, tais modos contribuem mais para eventos secos do que para eventos chuvosos, ao contrário do que ocorre nos dados observados (PREC_LA) (Tabela 2, Figuras 3c, 3d). Na banda interanual, tanto o Modo PR como o modo Inter contribuem mais para os eventos secos do que para os chuvosos, também ao contrário dos dados observados.

Para os dados PREC_FU (Tabela 2, Figuras 3e, 3f) os modos Sul e PR na banda sinótica contribuem mais para eventos secos do que para eventos chuvosos, semelhantemente aos dados observados do clima presente. Contudo, na banda intrassazonal, o Modo Sul contribui mais para os eventos secos, enquanto o Modo PR contribui mais para os eventos chuvosos, o que não concorda totalmente com os dados observados nem com os simulados do clima presente. Mesmo comportamento não alinhado totalmente com nenhum dos conjuntos de clima presente (observado e simulado) mostra a banda interanual, na qual o Modo PR contribui mais aos eventos chuvosos, enquanto o Modo Inter contribui mais aos eventos secos.

Observando-se a série de dados de clima futuro, para a escala sinótica, notou-se que ocorrem mais eventos curtos, principalmente de dois dias e, analisando os desvios de precipitação, notou-se que os desvios muito altos estavam relacionados a eventos "extremos", com valores muito altos em apenas um dia. Isto pode sugerir a diminuição dos eventos de chuva no futuro, mas não a intensidade dos mesmos. Estes resultados concordam com o observado por Marengo et. al. (2007), que mostram tendência de aumento na intensidade de eventos de chuvas extremas no período de 1960/90.

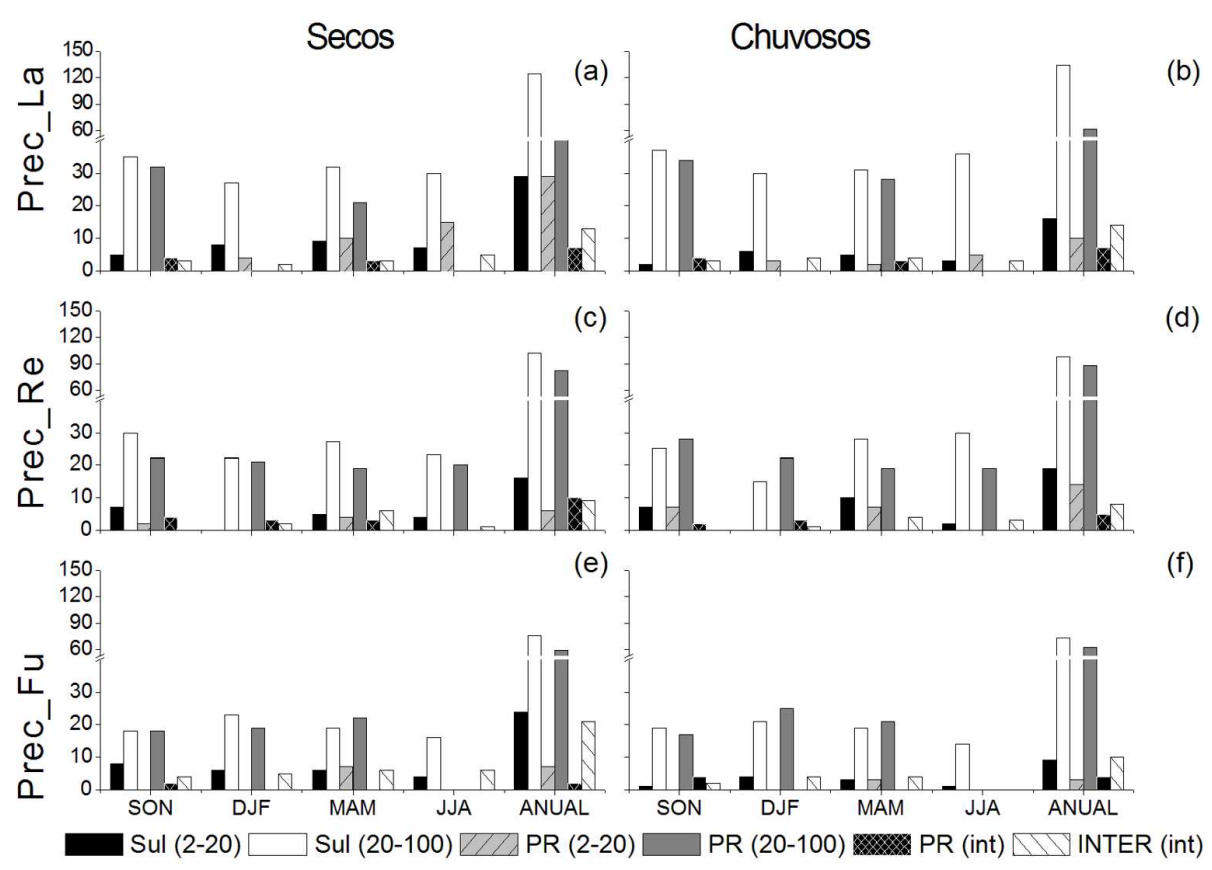

Figura 3: Eventos secos e chuvosos acumulados para cada estação do ano e somatória total de eventos no ano para os três conjuntos de dados e para cada banda de frequência (Sinótica 2-20, intrassazonal 20-100 e Interanual Int). (a,b) representam os eventos secos e chuvosos para os dados PREC_LA; (c,d) representam os eventos secos e chuvosos para os dados PREC_RE (e,f) representam os eventos secos e chuvosos para os dados PREC_FU. 
Tabela 2 - Números de eventos por ano. Para as séries PREC_LA e PREC_RE a soma total de eventos foi dividida por 25 e para a série PREC_FU por 17. Valores em negrito indicam o maior número médio de eventos por ano em cada modo.

\begin{tabular}{ccccccc}
\hline PREC_LA & SUL 2-20 & SUL 20-100 & PR 2-20 & PR 20-100 & PR-INT & INTER-INT \\
\hline Secos & $\mathbf{1 , 1 6}$ & 4,96 & $\mathbf{1 , 1 6}$ & 2,12 & 0,28 & 0,52 \\
\hline Chuvosos & 0,64 & $\mathbf{5 , 3 6}$ & 0,40 & $\mathbf{2 , 4 8}$ & 0,28 & $\mathbf{0 , 5 6}$ \\
\hline PREC_RE & & & & & & \\
\hline Secos & 0,64 & $\mathbf{4 , 0 8}$ & 0,24 & 3,28 & $\mathbf{0 , 4 0}$ & $\mathbf{0 , 3 6}$ \\
\hline Chuvosos & $\mathbf{0 , 7 6}$ & 3,92 & $\mathbf{0 , 5 6}$ & $\mathbf{3 , 5 2}$ & 0,20 & 0,32 \\
\hline PREC_FU & & & & & & \\
\hline Secos & $\mathbf{1 , 4 1}$ & $\mathbf{4 , 4 7}$ & $\mathbf{0 , 4 1}$ & 3,47 & 0,12 & $\mathbf{1 , 2 4}$ \\
\hline Chuvosos & 0,53 & 4,29 & 0,18 & $\mathbf{3 , 7 1}$ & $\mathbf{0 , 2 4}$ & 0,59
\end{tabular}

*SUL 2-20 e PR 2-20 são o Modo Sul e PR, respectivamente, na banda sinótica. SUL 20-100 e PR 20-100 são o Modo Sul e PR na banda intrassazonal. PR-INT e INTER-INT são o modo $\mathrm{PR}$ e Inter na banda interanual.

\section{CONCLUSÕES}

A análise de tendência, para os dados observados e simulados pelo modelo RegCM3 para o período de 1982 a 2006, apresentou boa concordância entre si apenas na região sul do estado do Rio Grande do Sul. As simulações do Modelo RegCM3 subestimaram as tendências de precipitação: as tendências que para os dados observados tinham significância estatística, na maioria dos pontos para os dados simulados não são significativas. Os dados de clima futuro mantiveram a mesma tendência de precipitação do clima presente no sul do Rio Grande do Sul, enquanto em outras regiões há algumas discrepâncias, principalmente no norte/noroeste do Rio Grande do Sul.

$\mathrm{Na}$ análise dos modos de variabilidade de precipitação encontraram-se três modos que influenciam o regime de chuvas na Região Sul do Brasil: o Modo Inter, o Modo PR e o Modo Sul. O Modo Inter foi encontrado na banda interanual, o Modo PR foi encontrado nas três bandas de frequência e o Modo Sul foi encontrado nas bandas intrassazonal e sinótica. Estes modos de variabilidade afetam de maneira diferente as estações do ano e regiões. Por exemplo, o Modo Sul afeta a precipitação no Rio Grande do Sul principalmente durante a primavera, o mesmo ocorre com o Modo PR, só que este afeta o Paraná. Já o Modo Inter possui uma influência mais ampla sobre o Rio Grande do Sul e também Santa Catarina e Paraná.

De maneira geral, os resultados das simulações do clima presente (PREC_RE) comparados com os dos dados observados (PREC_LA) só foram boas no Rio Grande do Sul, levando em conta as tendências de precipitação. No caso da contribuição dos modos analisados para os eventos secos e chuvosos, o comportamento dos dados observados e simulados é diferente.
Para o clima futuro, observa-se uma tendência, em relação ao clima presente simulado, para maiores números de eventos secos para os modos Sul e PR nas bandas sinótica e intrassazonal, e o Modo Inter na banda interanual. Com isso, ressalta-se a preocupação com a manutenção de água nos reservatórios para o consumo humano e para a agricultura, que dependem de chuvas regulares e bem distribuídas territorialmente.

\section{AGRADECIMENTOS}

Ao Conselho Nacional de Desenvolvimento Científico e Tecnológico (CNPq, projetos: 506372/2010-3, 303851/2009), FAPERGS (Fundação de apoio a pesquisa do Rio Grande do Sul, projeto: 11/2033-8) e a Coordenação de Aperfeiçoamento de Pessoal de Nível Superior (CAPES) pelo apoio financeiro. Ao Centro Regional Sul de Pesquisas Espaciais (CRS/INPE) pelo suporte técnico e espaço físico oferecido.

\section{REFERÊNCIAS BIBLIOGRÁFICAS}

BACK, A. J. Aplicação de análise estatística para identificação de tendências climáticas. Pesquisa Agropecuária Brasileira, v. 36, n. 5, p. 717-726, 2001.

CARDOSO, A. O. Relações entre a TSM nos oceanos Attântico e Pacífico e as condições climáticas nas Regiões Sul e Sudeste do Brasil. 2005. 158 p. Tese (Doutorado em Meteorologia) - Universidade de São Paulo, São Paulo, 2005.

CERA, J. C.; FERRAZ, S. E. T. Caracterização da Precipitação no Estado do Rio Grande do Sul.In: Anais do II Encontro Sul Brasileiro de Meteorologia, Florianópolis - SC, 2007. CERA, J. C.; FERRAZ, S. E. T.; BENDER, F. D. Influência da Oscilação Decadal do Pacífico e as Mudanças no Regime 
de Chuva do Rio Grande do Sul. Revista Ciência e Natura Especial, Santa Maria-RS. v. 1, 6a Ed., pg. 317-320, 2009.

DUCHON, C. E. Lanczos filtering in one and two dimensions. Journal of Applied Meteorology, v. 18, p. 1016-1022, 1979.

FERRAZ, S. E. T. Variabilidade Intrasazonal no Brasil e Sul da América do Sul. São Paulo, 2004. Tese (Doutorado em Meteorologia) - Universidade de São Paulo, São Paulo, 2004.

FIRPO, M. A. F., Influências remotas das TSM dos oceanos Pacífico e Atlântico e da Oscilação Antártica na variabilidade climática interanual no Rio Grande do Sul e suas inter-relações. 186p. Tese (Doutorado em Meteorologia) - Instituto Nacional de Pesquisas Espaciais, São José dos Campos, 2012.

GRIMM, A. M. ; FERRAZ, S. E. T. ; GOMES, J. Precipitation anomalies in Southern Brazil associated with El Nino and La Nina events. Journal of Climate, v. 11, n. 11, p. 28632880, 1998.

GRIMM, A. M. The El Niño impact on the summer monsoon in Brazil: regional processes versus remote influences. Journal of Climate, v.16, p. 263-280, 2003.

GRIMM, A. M. Clima da Região Sul do Brasil. In: CAVALCANTI, I. F. A.; FERREIRA, N. J.; JUSTI DA SILVA, M. G. A.; SILVA DIAS, M. A. F.(org). Tempo e Clima no Brasil, p. 135-147, São Paulo: Oficina de Textos, 2009.

GRIMM, A. M.; ZILLI, M. T. Interannual variability and seasonal evolution of summer monsoon rainfall in South America. Journal of Climate, v. 22, n. 9, p. 2257-2275, 2009. DOI: $10.1175 / 2008 J C L I 2345.1$

HAYLOCK, M. R. et al. Trends in Total and Extreme South American Rainfall in 1960-2000 and Links with Sea Surface Temperature. Journal of Climate, v. 19, p. 1490-1512, 2006.

IPCC, 2007 [Intergovernmental Panel on Climate Change]. Climate Change 2007: The Physical Science Basis. Contribution of Working Group I to the Fourth Assessment Report of the Intergovernmental Panel on Climate Change [Solomon, S., D. Qin, M. Manning, Z. Chen, M. Marquis, K.B. Averyt, M. Tignor and H.L. Miller (eds.)]. Cambridge University Press, Cambridge, United Kingdom and New York, NY, USA, 996 p.

JAMES, I. N. Introduction to Circulating Atmospheres. Cambridge Atmospheric and Space Science, 1994, 422p.

KAYANO, M. T.; ANDREOLI, R. V. Variabilidade Decenal a Multidecenal. In: CAVALCANTI, I. F. A. Tempo e Clima no Brasil. São Paulo, Oficina de Textos, p. 373-383, 2009.
LIEBMANN, B.; ALLURED, D. Daily Precipitation Grids for South America. Bulletin of the American Meteorological Society, 2005.

MARENGO, J. A. Mudanças climáticas globais e seus efeitos sobre a biodiversidade. Ministério do Meio Ambiente. Brasília-DF, 2006.

MARENGO, J. A. Mudanças climáticas e seus efeitos sobre a Biodiversidade: Caracterização do clima atual e definição das alterações climáticas para o Território Brasileiro ao longo do Século XXI. Ministério do Meio Ambiente. Series Biodiversidade, No. 26, 2007.

MARENGO, J. A. Mudanças Climáticas: Detecção e Cenários Futuros para o Brasil até o Final do Século XXI. In: CAVAlCANTI, I. F. A., et. al. Tempo e Clima no Brasil. São Paulo, Oficina de Textos, p. 407-424, 2009.

MINUZZI, R. B. VIANELLO, R. L. SEDIYAMA, G. C. Oscilações climáticas em Minas Gerais. Revista Brasileira de Meteorologia. São Paulo, v. 25, n. 2, 2010.

PAEGLE, J. N.; MO, K. C. Alternating wet and dry conditions over South America during summer. Monthly Weather Review, v. 125, p. 279-291, 1997.

PAL, J.S., E. E. SMALL, E. A. ELTHAIR. Simulation of regional-scale water and energy budgets: representation of subgrid cloud and precipitation processes within RegCM. Journal of Geophysical Research, v. 105, p. 29579-29594, 2000.

PAMPUCH, L. A. Investigação do Modo Sul no clima presente e futuro no Rio Grande do Sul. Dissertação (Mestrado em Meteorologia) - Universidade Federal de Santa Maria, Santa Maria, 2010.

SABATINI, A. D. Índices de extremos climáticos de temperatura e chuva na América do Sul: clima presente e validação do modelo RegCM3. Dissertação (Mestrado em Meteorologia) - Universidade de São Paulo, São Paulo, 2008.

SANSIGOLO, C. A.; KAYANO, M. T. Trends of seasonal maximum and minimum temperatures and precipitation in Southern Brazil for the 1913-2006 period. Theoretical and Applied Climatology, v. 101, p. 209-216, 2010.

SIEGEL, S. Estatística não-paramétrica. Ed. McGraw-Hill, Ltda. São Paulo, 1975, 350 p.

SNEYERS, R. Sur l' Analise Statistique des Séries d' Observations. Nota técnica OMM 143. 192 p. 1975. 